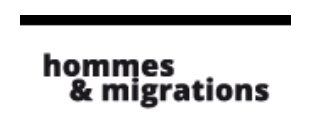

\section{Hommes \& migrations}

Revue française de référence sur les dynamiques

migratoires

$1317-1318$ | 2017

L'Europe en mouvement

\title{
« L'identité nationale toute seule ne suffit pas »
}

Entretien avec Enrico Letta, président de la Fondation Jacques Delors

\section{Marie Poinsot}

\section{(2) OpenEdition}

1 Journals

\section{Édition électronique}

URL : http://journals.openedition.org/hommesmigrations/3870

DOI : 10.4000/hommesmigrations.3870

ISSN : 2262-3353

\section{Éditeur}

Musée national de l'histoire de l'immigration

\section{Édition imprimée}

Date de publication : 1 avril 2017

Pagination : 53-56

ISBN : 978-2-919040-38-4

ISSN : $1142-852 X$

\section{Référence électronique}

Marie Poinsot, « «L'identité nationale toute seule ne suffit pas » », Hommes \& migrations [En ligne], 1317-1318 | 2017, mis en ligne le 01 avril 2020, consulté le 02 janvier 2020. URL : http://

journals.openedition.org/hommesmigrations/3870; DOI : 10.4000/hommesmigrations.3870 


\section{« L'IDENTITÉ NATIONALE TOUTE SEULE NE SUFFIT PAS »}

Entretien avec Enrico LETTA, président de la Fondation Jacques Delors, réalisé par MARIE POINSOT.

Hommes \& Migrations :

Pouvez-vous nous présenter en introduction l'Institut Jacques Delors et ses principales missions?

\section{Enrico Letta :}

L’Institut Jacques Delors a été fondé en 1996. Son rôle principal est d'animer le débat sur les politiques de l'Europe, en lançant des idées et des propositions en faveur de la construction européenne. Un deuxième volet de ses activités consiste à susciter des débats citoyens sur l'Europe et son futur. Ce sont les deux volets les plus importants de l'activité de l'institut que j'ai l'honneur de présider depuis un peu plus de six mois maintenant. Nous sommes actuellement investis sur un projet de « petite académie » qui donne la possibilité à ceux qui veulent les approfondir de s'approcher des thématiques européennes. Nous pensons qu'il est fondamental d'informer les citoyens qui agissent à l'intérieur de l'Europe car il y a un déficit dans la connaissance des institutions européennes, qui sont compliquées à appréhender. L'institut apporte ainsi sa petite contribution à une meilleure et plus large connaissance de l'Europe.

H\&M : Les migrations intra-européennes sont anciennes et ont même constitué l'essentiel des mouvements migratoires jusque dans les années 1960-1970, y compris après la Seconde Guerre mondiale. De nouveau, des Italiens, des Portugais, des Espagnols partent en France ou en Allemagne pour s'y installer. Pourquoi, d'après vous, cette histoire des mobilités intra-européennes est-elle si mal connue? Et comment l'inscrire davantage dans l'histoire européenne?

E. L. : Je pense que ce sont les migrations qui font l'histoire européenne. Des populations ont créé des liens encore plus forts entre nos différents pays. Des personnes se sont trouvées chez elles dans un autre pays. Elles y ont emmené une diversité d'empreintes culturelles, d'attitudes, de gastronomies, de religions. Cela a beaucoup aidé l'Europe. Aux pays fondateurs qui forment le noyau dur de l'Europe, se sont ajoutés l'Autriche, l'Espagne, le Portugal, la Grèce, puis d'autres pays au sein desquels le mélange des populations et la mixité des cultures sont très prononcés. C'est dans ces derniers pays que la mobilité a été la plus massive.

H\&M : Après la chute du mur de Berlin, n'y a-t-il pas eu une sorte de confusion au sein des pays européens sur le sens de l'Union et de la cohérence de la construction européenne?

E. L. : L'erreur commise à l'occasion de la chute du mur de Berlin a été de ne pas comprendre qu'il fallait créer une plus grande flexibilité ins- 
titutionnelle à l'intérieur de l'Europe. Au lieu de cela, s'est imposée l'idée selon laquelle il fallait ou bien être membre, ou bien être hors de l'Europe. C'est une situation que l'Europe est en train de payer aujourd'hui. On aurait pu tout de suite associer politiquement les pays de l'Est et envisager un parcours plus long du point de vue de leur intégration économique. Cela n’a pas été le cas. Je pense aussi que la question de la mobilité des Roumains ou des Polonais vers l'Italie, la France, le Royaume-Uni a joué également dans cette défiance vis-à-vis de l'Union. L'intégration des pays de l'Est sans flexibilité a créé de nombreux problèmes. Le référendum français de 2005 s'est joué sur l'image du plombier polonais. L'enjeu de la constitution européenne en a été complètement esquivé. La sortie du Royaume-Uni de l'Europe apparaît comme la conséquence tardive d'une gestion maladroite de cette construction européenne.

\section{H\&M : Alors que le débat sur les migrations extérieures à l'Europe reprend de l'ampleur, ne pensez-vous pas que, dans le projet politique de l'Union européenne, il faille non pas focaliser sur ces migrations externes vécues comme des menaces, mais sur les apports de la migration interne?}

E. L. : Ce sont deux choses très différentes, il faut donc les séparer. Désormais, l'Europe est un marché intérieur dans lequel il faut avoir des règles pour bien gérer les problèmes économiques. Il est inimaginable de retourner en arrière sur la question de la liberté de circulation et des migrations intra-européennes. Il faut considérer que nous sommes dans un espace dans lequel nous devons vivre cette mixité comme une grande richesse. Une autre chose est la migration externe aux frontières de l'Europe. C'est une question beaucoup plus complexe, pour les chiffres dont on parle, pour la diversité radicale de beaucoup de points de vue, et pour le futur de l'union. Sur une période très courte, l'Europe a reçu un million de réfugiés syriens. À ceux-là s'ajoutent, non pas des migrants économiques, mais tous les autres réfugiés qui fuient des guerres provoquées par les Américains, par exemple dans le cas des réfugiés irakiens ou afghans.

Le futur de l'Europe est lié au fait que ce continent est vieillissant. À l'inverse, l'Afrique va doubler sa population en 30 ans. Si ce continent continue à souffrir du manque de développement, la situation migratoire actuelle risque de se prolonger. Il faut donc que la politique migratoire de l'Union européenne se construise rapidement. Il s'agit d'un grand enjeu de politique interne et de stratégie pour le futur de l'Europe qui a besoin de migrants. Il y a des pays comme l'Italie, l'Allemagne, l'Espagne qui vont voir s'écrouler leur population active. Ce sera l'enjeu majeur pour les prochaines années. Il faut penser dès à présent au travail nécessaire pour mieux intégrer les immigrés dans les sociétés européennes.

\section{H\&M : Ce débat, délicat à introduire, demande de réfléchir sur la façon dont on intervient sur les imaginaires, sur les représentations, pas seulement sur les réalités sociales et économiques. Pensez- vous que l'Europe puisse se forger autour de l'idée d'une multiplicité culturelle et linguistique?}

E. L. : L'identité européenne ne doit pas effacer les identités nationales. Mais l'identité nationale toute seule ne suffit pas. Personnellement, je me sens italien mais je suis toscan et fier d'être de cette région. Je me sens en même temps fortement européen. C'est un sujet, de mon point de vue, essentiel et fondamental. Cette pluri-identité doit être introduite dès l'école. C'est la vraie différence par rapport à ce que j'ai vécu durant mon enfance. J'ai été à l'école en France et en Italie. Ce sont deux écoles qui ne se parlaient pas beaucoup. Chacune forgeait une identité nationale, d'un côté française, et italienne de l'autre. De plus, dans ma classe en Italie, il n'y avait que des Italiens, tandis que, dans ma classe en France, il n'y avait que des 
Strasbourgeois. Mes enfants, aussi bien dans leur classe à Rome qu'à Paris où ils vivent maintenant, sont dans des classes culturellement variées. Ce qui redéfinit la notion même d'identité nationale.

H\&M : L'euroscepticisme actuel n'est-il pas un frein à l'acceptation de ces migrations internes? Risquonsnous, de nouveau, des mouvements d'opinion hostile, non pas seulement à l'immigration externe, mais aussi interne, comme on peut l'observer avec le Brexit?

E. L. : C'est un sujet lié surtout à des réalités migratoires précises comme celles des travailleurs détachés. Il faudrait trouver des solutions sur ce sujet pour éviter de voir ces mouvements populaires prendre de l'ampleur dans les pays européens.

H\&M : En ce qui concerne l'évolution des migrations qualifiées intra-européennes envisagée à moyen et à long terme, des dispositifs comme les programmes Erasmus et les encouragements à la mobilité professionnelle peuvent-ils être un accélérateur des migrations internes? En quoi est-ce utile pour les marchés du travail nationaux et régionaux d'être très ouverts aux apports migratoires?

E. L. : L'Institut Jacques Delors a fourni un grand travail pour faire avancer cette idée de programmes comme Erasmus Pro qui favorisent l'apprentissage des adultes qui le souhaitent dans un autre pays européen. Le marché du travail européen doit se fixer des objectifs en matière de partage des outils et des compétences. Chaque pays de l'Union tente de favoriser ces migrations d'apprentissage et de formation professionnelle. Les règles fiscales changent, par les exemples que je connais comme en France, en Italie, au Portugal, dans le sens d'une plus grande attractivité.
H\&M : En tant qu'Italien, que notez-vous dans le débat en Italie sur cette nouvelle émigration italienne qualifiée?

E. L. : L'émigration italienne est absolument perceptible en Italie. Elle est la démonstration d'un problème national. Cette émigration est la conséquence d'un chômage des jeunes situé entre 37 et $40 \%$. Mais les jeunes ne sont pas les seuls touchés. Plusieurs domaines tels que l'université souffrent du manque d'attractivité de leurs activités. Toutes ces réalités sont en train d’appauvrir le pays, et plus particulièrement le Sud. Un travail de long terme sur la compétitivité du marché du travail italien est donc nécessaire.

H\&M : Plus généralement, on fête les 60 ans du traité de Rome. Est-ce que la liberté de circulation va dans le sens d'une fluidification du marché du travail européen ou est-ce qu'on voit encore des segmentations d'ordre national?

E. L. : Le marché européen reste très segmenté dans beaucoup trop de secteurs, comme le marché du travail et celui des capitaux. Vous avez des situations qui sont encore trop nationales. Leur fonctionnement est peu harmonisé et cela crée un tas de problèmes qui freinent la mobilité intra-européenne. Dans la mesure où les entreprises européennes cherchent à être dans un marché européen dynamique, le fait que les règles soient si différentes et compliquées n’est pas facile à accepter.

H\&M : Pensez-vous que la mémoire de ces migrations est préservée? L'enseignement de l'histoire de ces migrations permet-il de les valoriser? Quels sont les pays en pointe dans ce domaine? La préoccupation patrimoniale, mémorielle, de nos histoires communes et liées par ces migrations peut-elle permettre le développement du projet d'intégration européenne? 
E. L. : Vous touchez la question clé. Sans la connaissance de l'histoire et la compréhension d'où nous venons, il n'y a aucune capacité d'aller loin. J'en suis tellement convaincu que, chaque fois que je dois madresser en Italie à des publics très opposés à l'immigration, je lis toujours le texte de la commission du parlement du Congrès américain de 1915 sur l'immigration italienne. Sans dire d'où je le tiens, on pourrait dire qu'il a été écrit par un Italien pour décrire la situation des réfugiés syriens aujourd'hui dans les villes italiennes. Les gens sont stupéfaits quand ils se rendent compte que ce texte parle des Italiens aux États-Unis il y a plus d'un siècle. Quand on prend de la hauteur, quand on regarde l'évolution des choses, on a un tout autre point de vue. Moi aussi j'ai connu cette situation d'immigration. J'ai vécu à Strasbourg dans les années 1970 parce que mon père était professeur de mathématiques à l'université. J'y ai vu ce qu'était l'immigration de maçons italiens en Lorraine et en Alsace. Je l'ai vécue parce que j'ai vu l'importance du travail que ces Italiens ont effectué, les difficultés qu'ils ont rencontrées pour être acceptés, et le processus positif auquel cela a pu finalement aboutir. 